\title{
Automatic Assessment of Macular Edema From Color Retinal Images
}

\author{
K. Sai Deepak* and Jayanthi Sivaswamy, Member, IEEE
}

\begin{abstract}
Diabetic macular edema (DME) is an advanced symptom of diabetic retinopathy and can lead to irreversible vision loss. In this paper, a two-stage methodology for the detection and classification of DME severity from color fundus images is proposed. DME detection is carried out via a supervised learning approach using the normal fundus images. A feature extraction technique is introduced to capture the global characteristics of the fundus images and discriminate the normal from DME images. Disease severity is assessed using a rotational asymmetry metric by examining the symmetry of macular region. The performance of the proposed methodology and features are evaluated against several publicly available datasets. The detection performance has a sensitivity of $100 \%$ with specificity between $74 \%$ and $90 \%$. Cases needing immediate referral are detected with a sensitivity of $100 \%$ and specificity of $97 \%$. The severity classification accuracy is $81 \%$ for the moderate case and $100 \%$ for severe cases. These results establish the effectiveness of the proposed solution.
\end{abstract}

Index Terms-Abnormality detection, diabetic macular edema, hard exudates, learning normal.

\section{INTRODUCTION}

$\mathbf{S}$ WELLING in the macular region of retina which is also known as macular edema, is a complication of the eye often leading to reduced capacity of vision. Diabetic macular edema (DME) caused due to diabetes is a high risk complication which can cause irreversible loss of vision [1]-[3]. Early detection of even a minor sign of DME is essential as it may also appear without any external symptoms [4]. Once detected during retinal examination, it demands immediate treatment ranging from glycemic and blood pressure control, to laser surgery.

DME is generally detected directly or indirectly. Direct ways are using stereoscopy (for manual examination) or optical computed tomography images [3]. Indirect method is by detecting the presence of hard exudates (HE) in the retina. HE are formed due to secretion of plasma from capillaries resulting from the complications of retinal vasculature and could lead to retinal swelling. In color fundus images they appear as yellow-white deposits (see Fig. 1). Detecting the presence of hard exudates (HE) in different areas of retina is now considered a standard method to assess DME from color fundus images [1], [4], [5].

Manuscript received September 14, 2011; revised November 24, 2011; accepted November 26, 2011. Date of publication December 08, 2011; date of current version March 02, 2012. Asterisk indicates corresponding author.

*K. Sai Deepak is with the Center for Visual Information Technology, International Institute of Information Technology, Hyderabad, AP, 500032 India (e-mail: sai.deepak@research.iiit.ac.in).

J. Sivaswamy is with the Center for Visual Information Technology, International Institute of Information Technology, Hyderabad, AP, 500032 India (e-mail: jsivaswamy@iiit.ac.in).

Digital Object Identifier 10.1109/TMI.2011.2178856

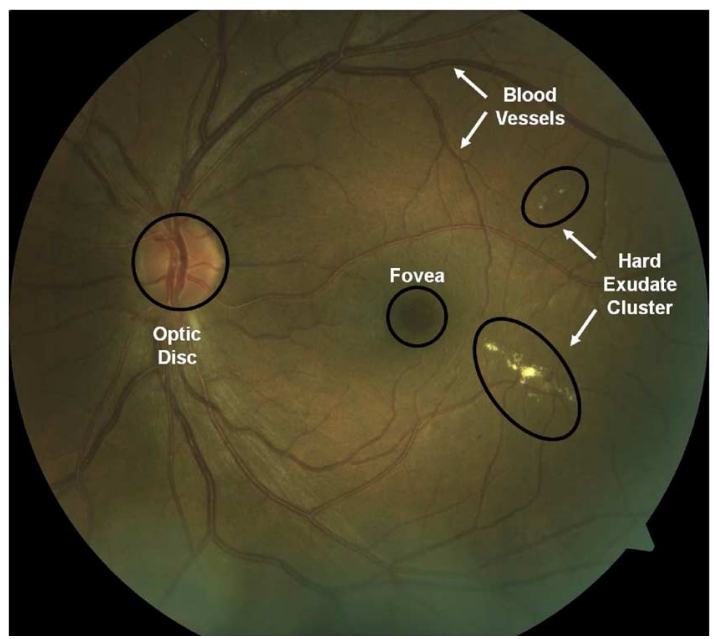

Fig. 1. Color fundus image with anatomical structures and lesions annotated.

The severity of the risk of edema is evaluated based on the proximity of HE to the macula, which is defined to be a circular region centered at fovea and with one optic disc (OD) diameter (see Fig. 1). The risk for DME increases when the HE locations approach the macula, with the risk being the highest when they are within the macula. This is an important factor in DME assessment for further referral of the patients to an expert.

Diabetes can also cause other retinal complications all of which are collectively termed as diabetic retinopathy (DR). Given the potential for vision loss and blindness due to DR, screening programs have been launched in many countries and color fundus image forms the basis for manual assessment in screening. Such manual assessment however is not scalable in large-scale screening scenario, particularly in developing countries either due to the scarcity of skilled manpower or unavailability of high end imaging equipment at the point of care. Solutions such as telescreening using permanent and mobile units to enable screening of retinal disorders in remote areas have been proposed [6], [7].

In such a scenario, an automatic disease detection system can significantly reduce the load of experts by limiting the referrals to those cases that require immediate attention. The reduction in time and effort will be significant where a majority of patients screened for diseases turn out to be normal. The ratio of normal patients to the ones showing disease symptoms can be as high as 9 to 1 in DR screening [8]. Several attempts have been reported towards building an automated solution for DR detection [8], [9]. Motivated by these attempts, we aim to develop a solution for automatic assessment of DME from color fundus images. Such a solution will be a value addition to the existing infrastructure of DR screening. 


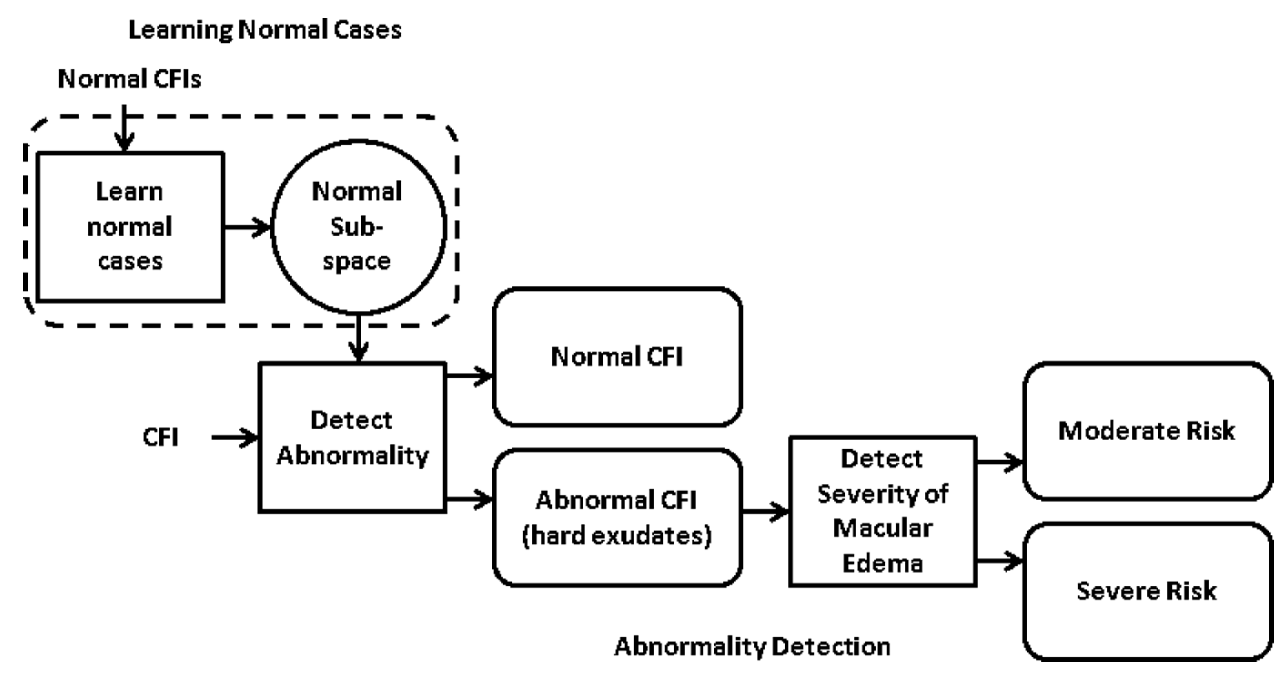

Fig. 2. Processing pipeline for detection and assessment of DME (CFI—color fundus image).

In order to develop a solution for automatic DME assessment, first a decision module is required to validate the presence or absence of HE in a given color fundus image. Once their presence is confirmed, a second module has to assess the macular region for measuring the risk of exhibiting DME. Therefore, in this work, we propose a two-stage methodology for detection and assessment of DME. The next section provides an overview of the earlier work carried out for detecting the presence of HE followed by an outline of the proposed methodology.

\section{PAST WORK}

Among recent approaches to direct detection of edema from color fundus images, multiple uncalibrated fundus images have been used to estimate a height map of macula in [2]. The estimated height map is generally noisy. A difference in the mean height is demonstrated between the height maps of normal and edema cases on synthetic images and only four sets of color fundus images. This difference is used to determine normal and abnormal cases. While the proposed method is encouraging, it requires more rigorous validation.

Using monocular color fundus image for detecting DME indirectly is still considered a reliable method in DR screening. Detecting DME is also done indirectly by detecting the presence of $\mathrm{HE}$ in images [5]. Automated solutions following this approach can be categorized as: 1) local schemes that perform localization of HE or HE clusters [10]-[23] and 2) global schemes for detecting the presence/absence of HE in images [1], [6], [24], [25].

\section{A. Local Schemes}

Given the relatively high contrast between the HE and retinal structures (except optic disc), the most common approach has been to process the green channel of the color fundus image and thresholding (fixed or adaptive/dynamic) the intensity histogram [12], [17], [22], [26]. Background suppression is another approach that has been used for finding HE candidates. Techniques explored for background estimation include median filtering [27], morphological operations [19], and clustering [11].
These approaches are sensitive to illumination changes that arise due to imaging conditions as well as changes in tissue pigmentation. This is addressed via an intensity normalization as a preprocessing step and/or removal of false positives with postprocessing in the above methods.

The well-defined edges of HE has also been used as a cue to identify candidate pixels. However, small or faint HE detection is difficult and hence additional rules are used to handle them [21]. [19], [20] also use edge properties in conjunction with other features to classify the detected candidates. In general, edge detection yields noisy results and hence preprocessing and postprocessing steps are required to reduce the large number of false candidates.

The distinct bright yellow color of HE has been the motivation for using color features [13], [15], [22]. Even though the use of color seems to be sufficient in principle, high variation in color witnessed in images across and within different ethnicities require a color and luminosity normalization step before detection.

The purpose of all the local schemes described here is to successfully segment or localize the exudate clusters with high accuracy so as to enable further assessment. As a result, several normal pixels are also detected as candidates in normal images. This affects the overall specificity of the system at image level HE detection.

\section{B. Global Schemes}

While the goal of local schemes in applying these approaches is to detect maximum number of HE, the global schemes relax this and try to ensure that at least the brightest pixels corresponding to HE in the image are detected. On these lines, [1] perform an initial candidate detection using a fixed threshold after a background subtraction step on the green channel image. This is followed by assignment of confidence values to every candidate based on the edge strength at that location. The confidence map is thresholded to obtain the final HE. A given image 
is considered to exhibit DME if at least one candidate is detected in the image. As observed with edge based methods earlier, a preprocessing step is required to avoid detection of a large number of non-HE edges.

Features such as visual word/group using a dictionary have also been used to represent color fundus images to help classify them as normal or abnormal [24], [25]. SIFT features of keypoints representing the HE candidates [24] and multiscale AM-FM features [25] have been used to construct the visual words. The visual word-based approach is robust to illumination changes across images. Creation of visual vocabulary requires clustering local regions into words/groups, with maximum discrimination between the background and HE regions. This is achieved by using a large number of clusters [24], [25]. The sensitivity of SIFT to local intensity variations results in detection of large number of false candidates which significantly increases the computational complexity of the overall detection.

Thus for DME detection, the strength of local schemes is the ability to detect small HE while the global schemes remove the burden of having to detect/segment every HE. We aim to explore using the global characteristics of an image while retaining the sensitivity to small HE. Towards this, we propose to transform the given image to an intermediate representation called motion pattern that spatially enhances the HE presence regardless of their size. This is followed by derivation of global features on the motion pattern for detection of HE.

Our strategy for detecting macular edema and evaluating its severity is as follows: the image under evaluation is first ascertained to be normal (abnormality detection) (see Fig. 2) by learning the characteristics of normal retinal images. Any deviation from normal characteristics is taken to be indicative of abnormality. For every abnormal image the severity of DME is assessed by determining the location of HE relative to the macula. In the next section, details of the proposed method is presented.

\section{PRoposed Method}

HE appear as clusters of bright, high contrast lesions and are usually well localized. The macula is a dark structure roughly at the center of the retina. In the absence of any HE (i.e., a normal retina), there is a rough rotational symmetry about the macula in the circular region of roughly twice the diameter of the optic disc.

We use this observation to derive relevant features to describe the normal and abnormal cases. Given a color fundus image, a circular region of interest (ROI) $I$ is first extracted and an intermediate representation $I_{\mathrm{MP}}$ also known as the motion pattern of the ROI is created. Relevant features are then derived for $I_{\mathrm{MP}}$ to classify the given image as normal or abnormal (containing HE).

\section{A. Region of Interest Extraction}

Since the severity of DME is determined based on the location of $\mathrm{HE}$ clusters relative to the macula, the images acquired for DME detection usually focus around the macular region. We find the best fit circle within the fundus mask [23] with macula at the center, for a given image. The region within this circle is the

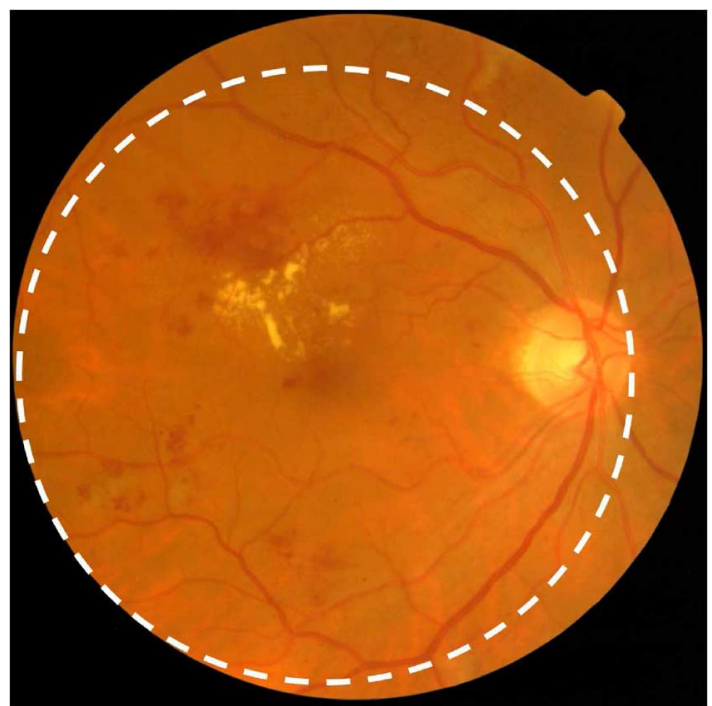

Fig. 3. Sample fundus image and the circular region of interest centered on macula.

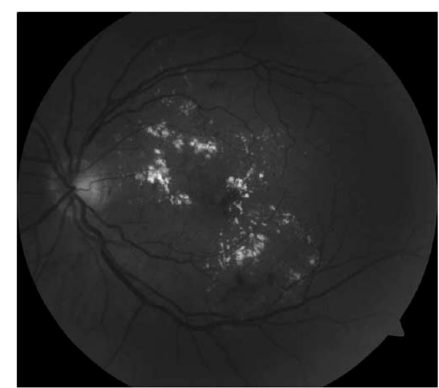

(a)

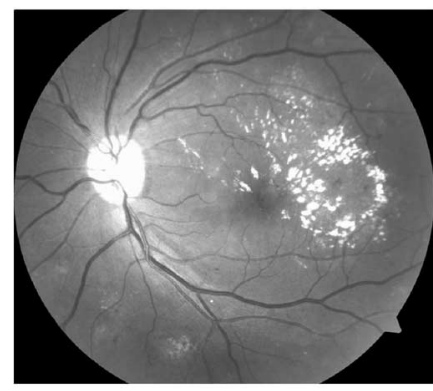

(c)

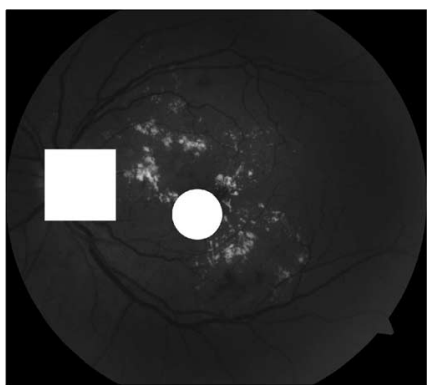

(b)

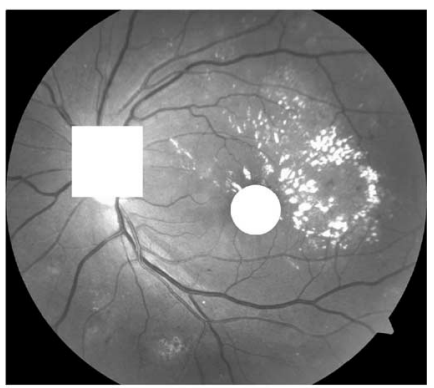

(d)
Fig. 4. Sample images (green channel) and result of macula and optic disk detection. OD is indicated by a bright rectangular mask and the macula location by a circular mask. (a) Sample image A. (b) Detected macula and OD for sample image A. (c) Sample image B. (d) Detected macula and OD for sample image B.

desired ROI denoted as $I$ (see Fig. 3 for an example). The green channel of $I$ forms the input for all subsequent processing.

The center of macula is automatically detected using [28] and restricting the search to a central region of the given image since the acquired images for DME detection are macula-centric. Since the OD shares a brightness characteristic similar to HE, it is also automatically detected and masked using [29]. The result of macula and optic disc detection can be seen in Fig. 4 


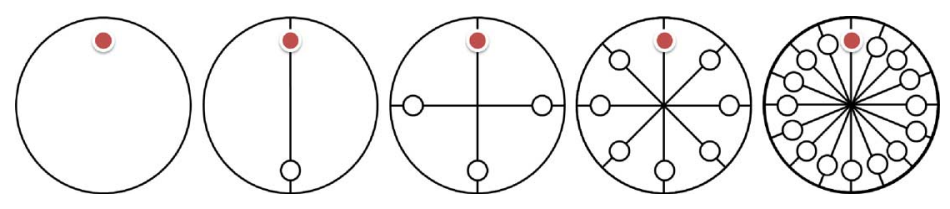

Fig. 5. Graphical depiction of motion pattern generation. First pattern: a disk with one lesion (shown as a red dot). The rest are results of applying motion with decreasing rotation steps: $\pi,(\pi / 2),(\pi / 4),(\pi / 8)$. In each case, the union of the patterns obtained after a complete cycle of rotation $(0$ to $2 \pi)$ is shown.

where the macula is shown as a circular patch and the OD is shown as a rectangular patch.

\section{B. Generation of Motion Patterns}

The creation of a motion pattern is motivated by the effect of motion on biological/computer visual system. These systems represent a scene as a set of spatially sampled (by the sensors/detectors) intensities or an image. This sampling is uniform in cameras while it is log polar in human eyes. When an object in a scene moves at a high speed, it usually leaves a smearing pattern in the captured image. Generally, the spatio-temporal changes recorded by the sensor are characteristic of the moving object [30]. In computer vision, the estimation and removal of the smear pattern, also popularly known as motion blur in images, has been an active area of research [31]. We argue that there is much information about the scene in the smear pattern and propose to use it to represent an image. We do this by simulating this operation in a single image by inducing motion.

Signal aggregation at sensor locations in human eyes [32] and camera, gives rise to the smearing effect. In order to simulate this effect, we induce motion in a given image to generate a sequence of images. These are combined by applying a function to coalesce the intensities at each sensor location to give rise to a motion pattern. Let the given ROI be denoted as $I(\vec{r})$. A motion pattern $I_{\mathrm{MP}}$ for $I$ is derived as follows:

$$
I_{\mathrm{MP}}(\vec{r})=f\left(G_{N}(I(\vec{r}))\right)
$$

where $\vec{r}$ denotes a pixel location, $G_{N}$ is a transformation representing the induced motion which is assumed to be rigid. Practically speaking, $G_{N}$ generates $N$ transformed images which are combined using $f$ to coalesce the sampled intensities at each pixel location (1). Here, $G_{N}(I)$ is expressed as follows:

$$
G_{N}(I)=\left\{R_{\theta_{n}}(I)\right\}
$$

where $R$ is a rotation matrix. The rotation angle $\theta_{n}=n \theta_{0}$ with $n=0,1, \ldots N ; \theta_{0}$ denotes the rotation step. When $n=0$, we have no rotation and hence $R_{0}(I)=I$. Thus $G_{N}$ is a set of rotated versions of the given $I$ and the total number of rotated images $N=(2 \pi) /\left(\theta_{0}\right)$. The sampling rate $\theta_{0}$ of the detector determines the number of images generated in the set $G_{N}$. In the problem at hand, since HE appear as bright localized lesions against the retinal background, they should form a bright smear pattern in $I_{\mathrm{MP}}$ whereas the textured background will be smoothed out. This representation can thus spatially enhance the characteristics of $\mathrm{HE}$ and help improve their detectability. At the same time, this should also serve to minimize the effect of the variability observed across images by smoothing them out.
Since, the severity of the disease is directly related to the radial distance of HE in the circular ROI, rotational motion is induced to generate the desired $I_{\mathrm{MP}}$. The transformation function $G_{N}$ is applied to $I$ to generate a sequence of $N$ images which are rotated versions of $I$. The spatial extent of smearing of intensities depends on the maximum rotation whereas the sampling rate at each location is directly related to the size of each rotation step. The generation of motion patterns is shown graphically in Fig. 5. Consider a disk with a single circle near the periphery modeling a lesion (first pattern). When rotation is applied to this pattern in steps of $\theta_{0}$, a set of patterns are generated. When $\theta_{0}=\pi$ two patterns will be generated and their union is the second pattern in Fig. 5. The remaining patterns in this figure are the result of the union of patterns generated with decreasing step size. It can be observed that a decrease in the step size results in several copies of the lesion in the final result. In this example, the motion pattern $I_{\mathrm{MP}}$ is obtained by using the union operation as the coalescing function. In practice, any coalescing function $f$ can be employed. The strength of the signal at $I_{\mathrm{MP}}(\vec{r})$ will not only depend on the choice of $f$, but also on the sampling rate $\theta_{0}$.

In the problem at hand, the choice of $f$ should ideally 1) enhance the HE by increasing the extent of the smear caused by it in the motion pattern and 2) increase the homogeneity of retinal background. Accordingly, two functions namely Mean and Maximum were considered in this work. These are defined as follows:

$$
\begin{aligned}
I_{\mathrm{MP}}^{\mathrm{mean}}(\vec{r}) & =\frac{1}{N} \sum_{n=0}^{N-1} R_{\theta_{n}}(I(\vec{r})) \\
I_{\mathrm{MP}}^{\max }(\vec{r}) & =\max _{n=\{0 \ldots(N-1)\}} R_{\theta_{n}}(I(\vec{r})) .
\end{aligned}
$$

While the coalescing function Mean (2) tries to achieve the averaging effect observed in motion blur, Maximum (3) tries to exploit the fact that HE usually appear brighter than any other structures in the background at the same radial distance. The original and motion pattern images in Fig. 6 illustrate the effect of the two coalescing functions on a normal and two abnormal fundus images. It can be seen that the motion patterns are clearly distinct for these two classes.

\section{Feature Selection}

The motion pattern $I_{\mathrm{MP}}$ generated by inducing motion on $I$ results in the smearing of lesions when present, along the motion path (as in Fig. 6). To effectively describe this motion pattern, we propose to use a descriptor derived from the Radon space. The Radon transform $P_{\alpha}$ of $f(x, y)$ is the integral of $f(x, y)$ 


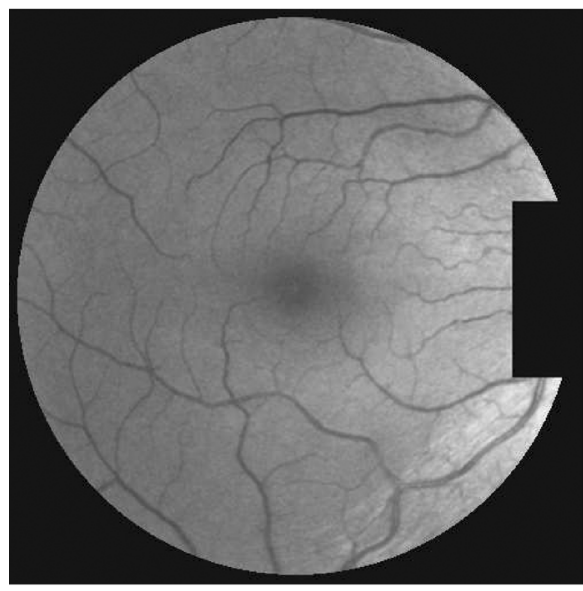

(a)

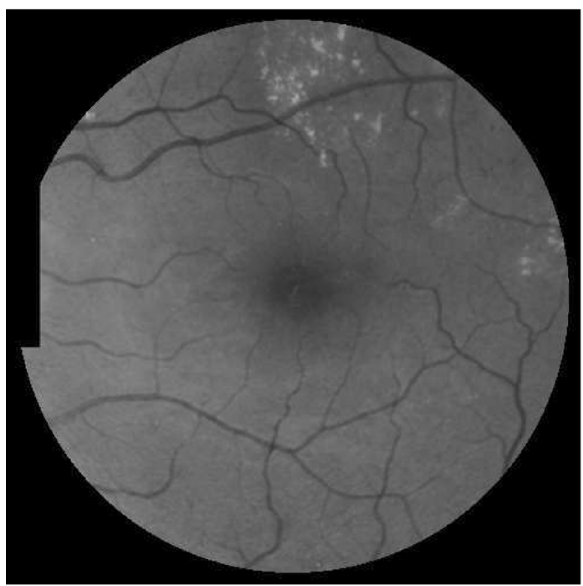

(d)

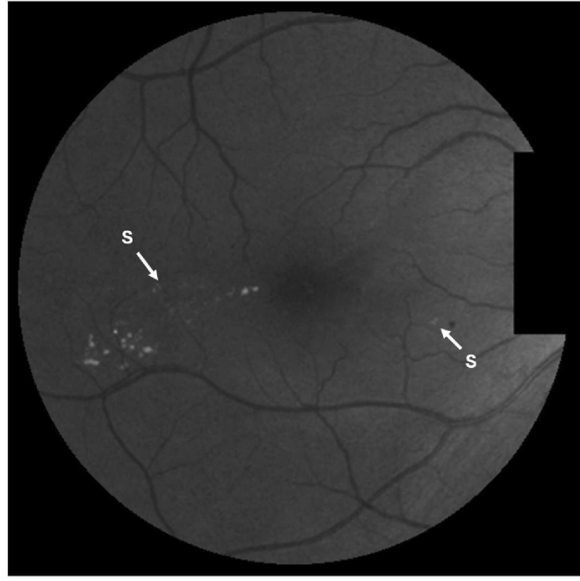

(g)

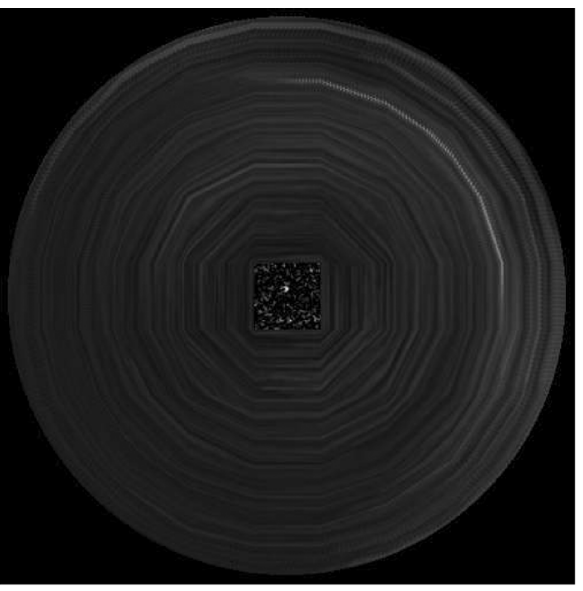

(b)

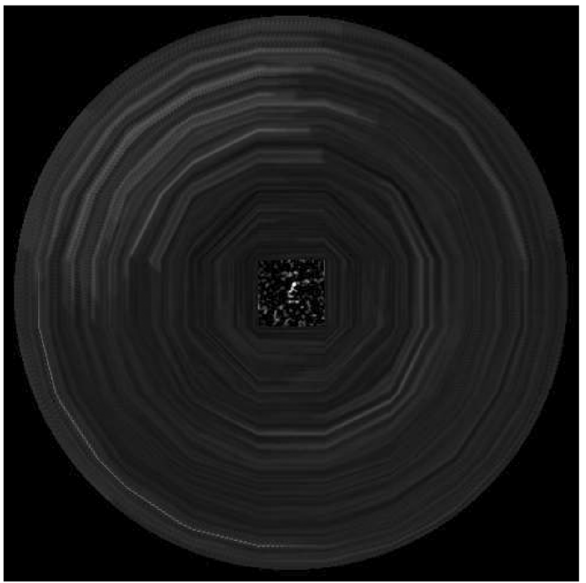

(e)

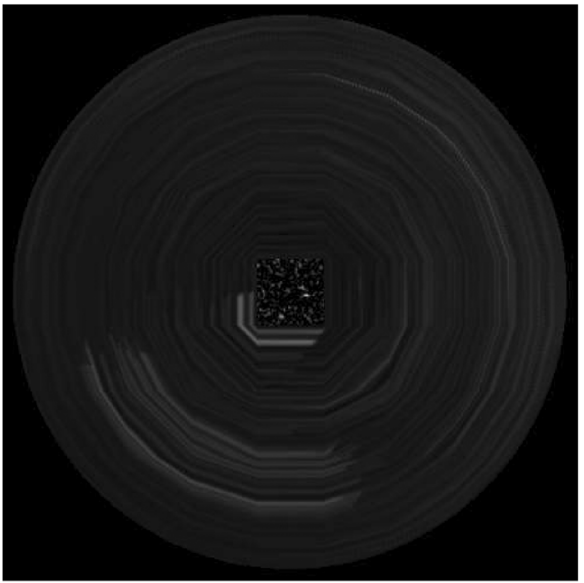

(h)

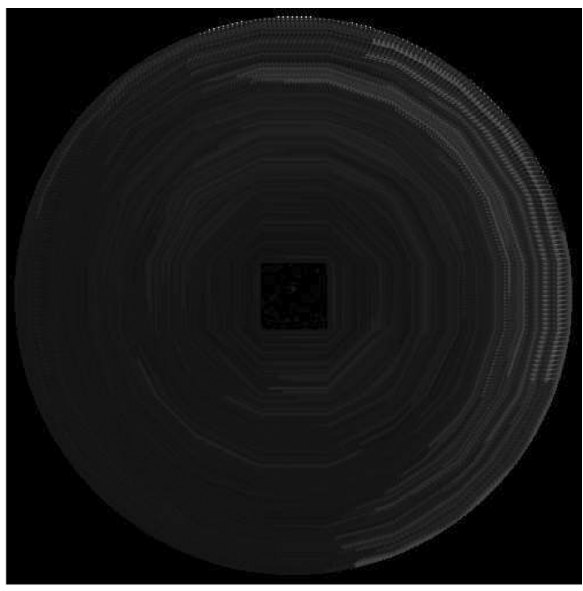

(c)

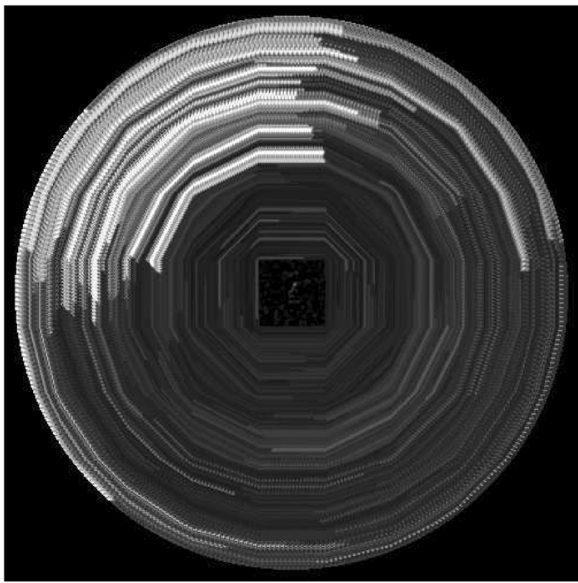

(f)

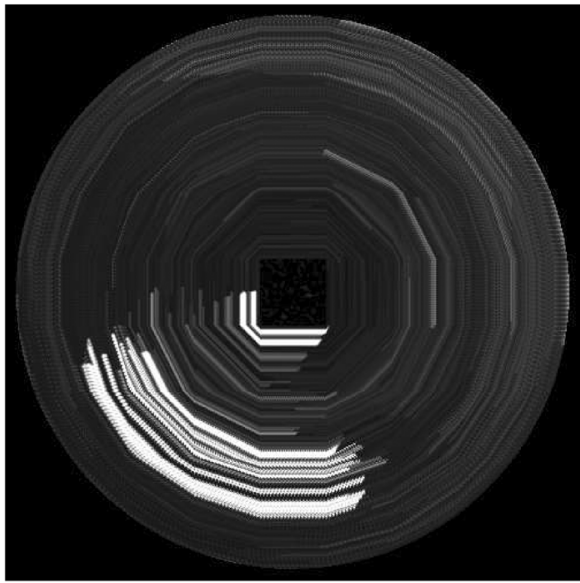

(i)

Fig. 6. Sample fundus images (column 1) and their motion patterns for the mean (column 2) and maximum (column 3 ) as coalescing functions. Samples belong to normal (top row), moderate (middle row) and severe (last row) DME cases. Rotation (anticlockwise) step $-1^{\circ}$ and maximum rotation $-90^{\circ}$. Annotation (S) indicates location of subtle (faint) hard exudates in color fundus image.

along a line oriented at $\alpha$ and distance $r$ from the origin. Given $f(x, y)$, its angular projection $P_{\alpha}$ is computed as

$$
P_{\alpha}(r)=\int_{-\infty}^{\infty} \int_{-\infty}^{\infty} f(x, y) \delta(r-x \cos \alpha-y \sin \alpha) d x d y
$$

$\alpha$ is the angle between the line and $y$ axis.
In our problem, the image $I_{\mathrm{MP}}$ is projected to obtain a vector response for every angle $\alpha$ and the desired feature vector then is derived by concatenating the responses $P_{\alpha}$ for different orientations. The spatial extent of any HE that may be present, is enhanced in the motion pattern $I_{\mathrm{MP}}$ and is in turn reflected immediately in the projection based feature vector. Thus, the feature vector for an abnormal retina will have several peaks in 
its profile due to intensities corresponding to HE. On the other hand, the feature vectors for a normal retina will have relatively uniform values resulting in a compact normal subspace. These feature vectors are used for learning the subspace corresponding to normal images.

\section{Abnormality Detection-Learning Normal Class}

Learning normal cases is achieved using single class classification. In this approach, a classification boundary is formed in the feature space around the subspace corresponding to normal cases. If a new image, when transformed to this feature space, lies within this boundary, then it is classified as normal and abnormal otherwise. Two simple single class classifiers are considered in this work: Gaussian data description (Gaussian DD) and principal component analysis data description (PCA DD).

Gaussian DD: Here, the normal class is modeled as a Gaussian distribution. The model parameters, namely, the mean $\mu$ and the covariance $\sum$ are computed for the training set made of normal cases. Classification of a new case is based on the Mahalanobis distance between the new case and the normal subspace which is computed as

$$
D\left(g\left(I_{\mathrm{MP}}\right)\right)=\left(g\left(I_{\mathrm{MP}}\right)-\mu\right)^{T} \sum^{-1}\left(g\left(I_{\mathrm{MP}}\right)-\mu\right) .
$$

$P C A D D$ : Here, a linear subspace is defined for the normal cases. The eigenvectors corresponding to the covariance matrix $\sum$ of the training set is used to describe the subspace. The feature vector $g\left(I_{\mathrm{MP}}\right)$ for a new case is projected to this subspace and again reconstructed $g\left(I_{\mathrm{MP}}\right)_{\text {proj }}$. The new case is classified to be normal based on a reconstruction error defined as

$$
e\left(g\left(I_{\mathrm{MP}}\right)\right)=\left\|g\left(I_{\mathrm{MP}}\right)-g\left(I_{\mathrm{MP}}\right)_{\mathrm{proj}}\right\|^{2} .
$$

For both the above single class classifiers, the classification between normal and abnormal images is then performed using an empirically determined threshold on $D\left(g\left(I_{\mathrm{MP}}\right)\right)$ and $e\left(g\left(I_{\mathrm{MP}}\right)\right)$ for Gaussian DD and PCA DD classifiers, respectively. Thus far, we have described the methodology for determining if a given image is normal or abnormal. Next, we will present a solution for further subclassifying an abnormal image according to the severity of DME.

\section{E. Determining the Severity of Macular Edema}

Assessing the severity of macular edema is the next task. Here, the macular region which is the circular ROI within 1 optic disc diameter from the center, is of key interest as any HE within this region indicates high risk for DME, requiring immediate attention. The macula in a normal image is relatively darker than other regions in the fundus image and is characterized by (rough) rotational symmetry. We use this symmetry information to establish the risk of exhibiting edema: good degree of symmetry is taken to indicate the abnormality is not inside macula and hence it is declared as a moderate case. Asymmetry of the macula on the other hand implies abnormality is within the macula and hence the case is deemed severe. A method to detect severity of edema based on rotation symmetry has also been used earlier where the symmetry of larger ROI centered on the

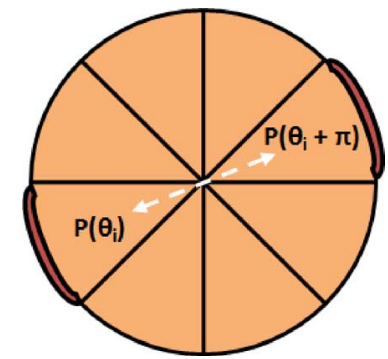

Fig. 7. Illustration of using rotational symmetry of macula for describing severity of edema.

macula is considered [33]. We propose a slightly different approach by considering the symmetry of only the macular region. Unlike [33], removal of blood vessels is not required here, as visibly large vasculature is usually not observed within macula in color fundus image.

A symmetry measure $S$ is defined as the second norm of the distance between the histograms of diametrically opposite pair of patches $\left(P\left(\theta_{i}\right)\right.$ and $\left.P\left(\theta_{i}+\pi\right)\right)$. In our work, eight angular samples were used to create eight patches $\left(P_{i}\right)$ from the circular ROI as illustrated in Fig. 7 and a histogram of 10 bins was computed for every patch. Since the intensities corresponding to HE contribute mostly to the higher bins in the histogram, only the last five bins are used for measuring the symmetry. A preprocessing step was performed to eliminate any intensity bias as in [33].

A threshold on the symmetry measure $S$ is used for assessing the degree of abnormality of an image as moderate or severe risk of DME. Let $S_{\max }$ and $S_{\min }$ be the maximum and minimum symmetry values for normal images in the training set used for abnormality detection. Then the severity of a given abnormal image $I_{a}$ is determined by comparing the symmetry measure of this image $S\left(I_{a}\right)$ against a threshold $T$ as follows:

$$
\text { Severity }\left(I_{a}\right)= \begin{cases}\text { moderate, } & \text { if } S\left(I_{a}\right) \leq T \\ \text { severe, } & \text { otherwise }\end{cases}
$$

It is desirable to set the threshold $T$ to be a percentage $p$ of the maximum symmetry value for normal images. Hence, the threshold is selected as

$$
T=p\left(S_{\max }-S_{\min }\right)+S_{\min } .
$$

This definition for $T$ permits the value of $p$ to be in [0-1].

It is desirable to select a low value for $p$ to achieve highest classification accuracy for the severe class of DME images as they require immediate medical attention. Next, we describe the assessment for the proposed DME detection method.

\section{EXPERIMENTS AND RESULTS}

The experiments for assessing the proposed method were performed on four publicly available datasets of color fundus images. A set of experiments were first conducted to determine the optimal motion parameters. Next, using these results, the method was assessed on the datasets and compared with existing algorithms for DME detection. 


\section{A. Dataset}

Four publicly available datasets were used for validating the proposed method. The first two of the four datasets described next have associated annotations at image level for DME.

The DMED or HEI-MED dataset [1] comprises of compressed (Jpeg) images where 68 fundus images not exhibiting any signs of a disease whereas 54 fundus images contain $\mathrm{HE}$. These images are acquired from patients of different ethnicity and age group. Consequently, a large variability is observed across images in color and tissue pigmentation. 18 (of the 68) normal images were randomly selected and used for creating the normal subspace while the remaining images were used for testing the performance of the method. The abnormal images with no signs of hard exudates were excluded from the experiments.

The MESSIDOR dataset [34] comprises of macula centered color fundus images, acquired at multiple resolutions, of patients showing multiple symptoms of DR. 400 images were used for validation of the proposed method. Of the 400 images, 274 are normal and 126 are abnormal images and contain HE. 41 of these abnormal images are annotated as showing moderate severity of macular edema while the rest of 85 images are annotated to be severe cases. All the abnormal images are acquired at Service d'Ophtalmologie-Hopital Lariboisiere Paris. For our work, the normal subspace was created using features from 74 normal images randomly selected from the dataset.

Two popular datasets Diaretdb0 [35] and Diaretdb1 [36] were also used for validating the proposed method. The Diaretdb0 dataset contains 20 normal color fundus images and another 59 images showing at least the signs of hard exudates. The $D i$ aretdb1 database consists of five normal color fundus images and 38 images with the signs of hard exudates. As the number of normal and abnormal images in either of the datasets are few, a ten-fold cross-validation is performed for performance analysis.

\section{B. Motion Parameter Estimation}

The discriminability between normal and abnormal retinal images is contingent on the right choice of parameters when inducing motion in the static image. Two parameters govern the rotational motion: the rotation step (in degrees) which controls the sampling rate at each location and the function $f$ used for coalescing the samples. In order to determine the optimal parameters, we conducted a set of experiments.

A normal retinal image was created by averaging the green channel of 400 retinal images from MESSIDOR dataset. No preprocessing was performed in order to retain all variations in intensity bias that occur in practice. Only a circular region of interest centered at macula and with radius of two optic disc diameter was used in the experiments for simplicity. An abnormal retinal image was synthesized from the averaged normal retina by adding a localized circular structure with high contrast, to model an HE lesion [see Fig. 8(a) and (b)]. The local contrast of lesion in Fig. 8(b) can be seen at $75 \%$ of the retinal background. This procedure permits varying lesion properties (contrast and size) and choosing motion parameters that provide maximum class discriminability between normal and abnormal images.

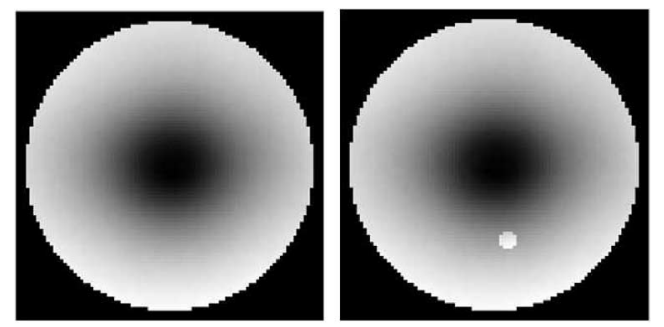

(a)

(b)

Fig. 8. Representative normal and abnormal retina. The abnormal retina is modeled by adding a bright lesion to emulate HE. (a) Normal retina. (b) Abnormal retina.

Rotational motion was induced on the modeled normal and abnormal images using both mean and maximum as the coalescing function. Presence of lesion in the abnormal image results in smearing of lesion intensities over the motion path in the motion pattern. This effect of lesion on retinal background can be observed as change in local information with respect to the motion pattern of normal retina. Given a motion pattern $I_{\mathrm{MP}}$ let its gradient (magnitude) be denoted as $\nabla I_{\mathrm{MP}}$. The local (Shannon's) entropy $H(Y)$ at every point $Y$ in this gradient image is computed over a local neighborhood $\Omega$ of $Y$ to create an entropy map. The total entropy is computed by summing the entropy at every point $Y$ to yield a measure of the disturbance in local information

$$
H_{\mathrm{MP}}=\sum_{Y} H\left(\nabla I_{\mathrm{MP}}(Y)\right) .
$$

In our simulation, the size of $I_{\mathrm{MP}}$ was $150 \times 150$ and the $\Omega$ was a neighborhood of size $7 \times 7$. A measure $d$ for the discriminability of normal and abnormal retinal images is then defined as the absolute difference between the entropy values for normal and abnormal images

$$
d=\left|H_{\mathrm{MP}}^{\text {abnormal }}-H_{\mathrm{MP}}^{\text {normal }}\right| .
$$

1) Effect of Rotation Step and Coalescing Function on Class Discriminability: A set of plots of $d$ as a function of motion parameter (rotation step) and lesion size and contrast are shown in Fig. 9 for one complete rotation and for two choices for $f$ the coalescing function. It can be observed that the discriminability (d) decreases as the rotation step is increased for the maximum coalescing function [see Fig. 9(a)]. This effect is apparent from the fact that the lesion is a high contrast structure. In the case of mean it is interesting to note that the discriminability initially increases slowly with increasing rotation step followed by a decrease. It is essentially due to the fact that for smaller rotation steps a large number of samples are accumulated at a location resulting in the reduction of contrast of lesion in the motion path (or the smear pattern). Whereas for a large rotation step, the smoothing effect observed in the retinal background for mean reduces and eventually contributes to the local disturbance measure. Hence, we conclude from this simulation that a maximum coalescing function and rotation step of around $1^{\circ}$ yield highest discriminability between normal and abnormal images.

2) Effect of Lesion Size and Contrast on Class Discriminability: Next, we observe the effect of lesion size as the 


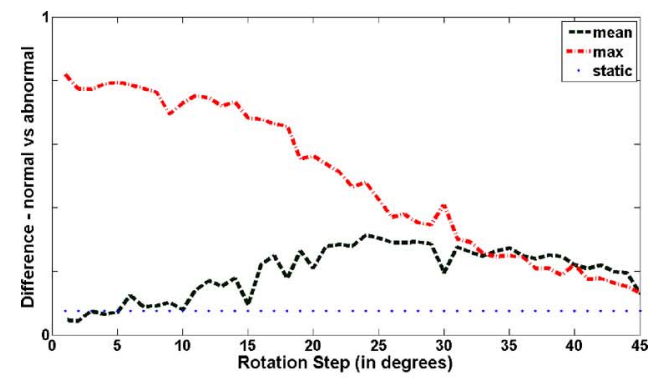

(a)

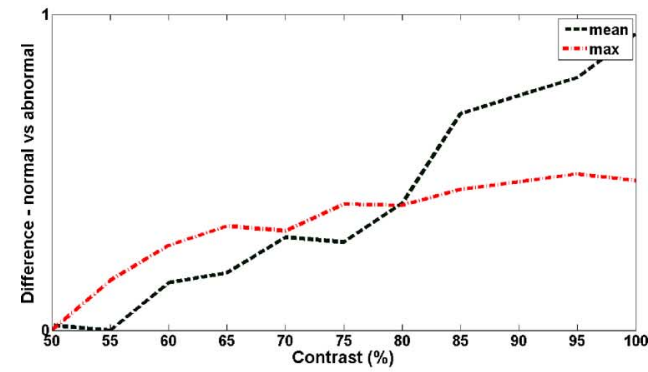

(b)

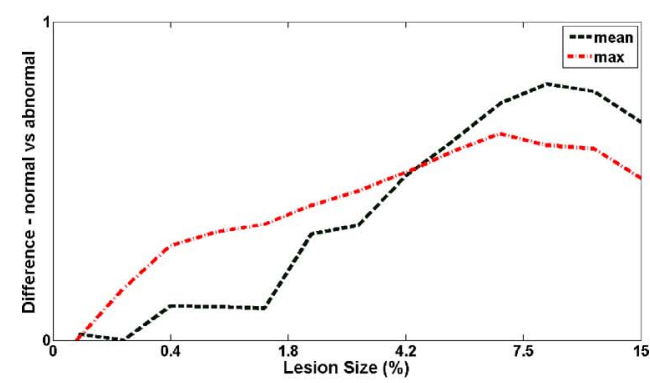

(c)

Fig. 9. Effect of increasing rotation step, lesion contrast, and lesion size on abnormal retina for estimating motion parameters. (a) Effect of rotation step on $d$. (b) Effect of local contrast on $d$. (c) Effect of lesion size on $d$.

percentage of ROI (resolution) and lesion contrast with respect to the retinal background for the selected rotation step and the coalescing function. This will help us to analyze the effect of lesion and image properties on the discriminability $(d)$ between the normal and abnormal class and also validate the selection of motion parameters.

As HE clusters also appear at various contrast and sizes, we observe the effect of changing these two parameters by fixing the rotation step to $1^{\circ}$. The values for contrast and lesion sizes are varied based on the observation of HE clusters in MESSIDOR. The discriminability is higher initially for the maximum rather than the mean coalescing function for low lesion contrast, but this effect is reversed as the lesion contrast increases [see Fig. 9(b)]. Similar behavior is also observed with increasing lesion size on discriminability of normal and abnormal retina [see Fig. 9(c)]. Based on these observations, we conclude that using maximum as coalescing function enables better detection of lesions even when they are small and have low local contrast. Therefore the suggested motion parameter values can be used on retinal images irrespective of the size and resolution of the ROI selected.

\section{Detection of Macular Edema}

Based on the above experimental findings, the motion parameters and coalescing function were chosen for the assessment of the proposed method. The coalescing function of maximum was chosen and three rotation step sizes, $1^{\circ}, 3^{\circ}$ and $5^{\circ}$ were considered. Since the optic disc is also a bright, albeit large structure, it was masked out before inducing motion. The result of inducing motion on an abnormal ROI with a quarter cycle of rotation $\left(90^{\circ}\right)$ and rotation step of $1^{\circ}$ can be seen in Fig. 6 . The effect of using the mean and maximum coalescing functions on the normal fundus image can be seen in the top row of Fig. 6 . Similarly, motion patterns for images with risk of DME can seen in the middle row of Fig. 6 for moderate case and in the last row of Fig. 6 for the severe case. For generating motion pattern with mean as the coalescing function, the masked region corresponding to optic disc was filled using nearest neighbor interpolation scheme to maintain a circular ROI. It can be observed that even the smallest and faintest HE lesions in Fig. 6(g) leave distinct smear patterns [see Fig. 6(i)]. Since the objective of this work was to evaluate disease detection performance, detection of anatomical locations in retina, like macula and optic disc center were not automated.

A descriptor based on Radon transform described earlier was computed with a resolution of $30^{\circ}(M=6)$ with six bins each, creating a compact feature vector of dimension 36 .

The Gaussian DD and PCA DD classifiers were trained on the normal subspace using the feature vectors on the training set of normal images. For Gaussian DD, the mean and the covariance were computed from the feature vectors corresponding to normal training images. A threshold on the Mahalanobis distance $D\left(g\left(I_{\mathrm{MP}}\right)\right)$ was used for separating the normal and abnormal classes. In the case of PCA DD, the feature vectors were projected onto six dimensions to compute the reconstruction error $\left(e\left(g\left(I_{\mathrm{MP}}\right)\right)\right)$. Normalized threshold ranging from 0 to 1 was applied on the parameter $D\left(g\left(I_{\mathrm{MP}}\right)\right)$ and $e\left(g\left(I_{\mathrm{MP}}\right)\right)$ to generate receiver operating characteristic (ROC) curves for both the classifiers. Here, sensitivity indicates the successful detection of images containing HE whereas specificity corresponds to correctly identifying normal images.

1) ROC Plots: The ROC plots are presented in Fig. 10 for the DMED dataset. From Fig. 10(a) it can be observed that the Gaussian distribution assumption for the normal DMED images is incorrect as it results in a low specificity of around 50\% at $100 \%$ sensitivity for HE detection. The performance is improved with PCA DD classifier where a specificity of $74 \%$ is achieved at $100 \%$ sensitivity and the area under the ROC curve (AUC) is 0.99 . These figures are a significant improvement over the detection accuracy reported in [1] (see Table I) which also uses a global approach.

Fig. 11(a) and (b) depicts the ROC curves for the MESSIDOR dataset using Gaussian DD and PCA DD classifiers respectively. The plots indicate that the performance of both classifiers are on par due to the fact that the number of images is higher in this dataset (than in DMED) and is better distributed. The best performance is once again achieved with the PCA DD classifier. The Table II tabulates performance figures which indicate that the proposed method has an improved performance 


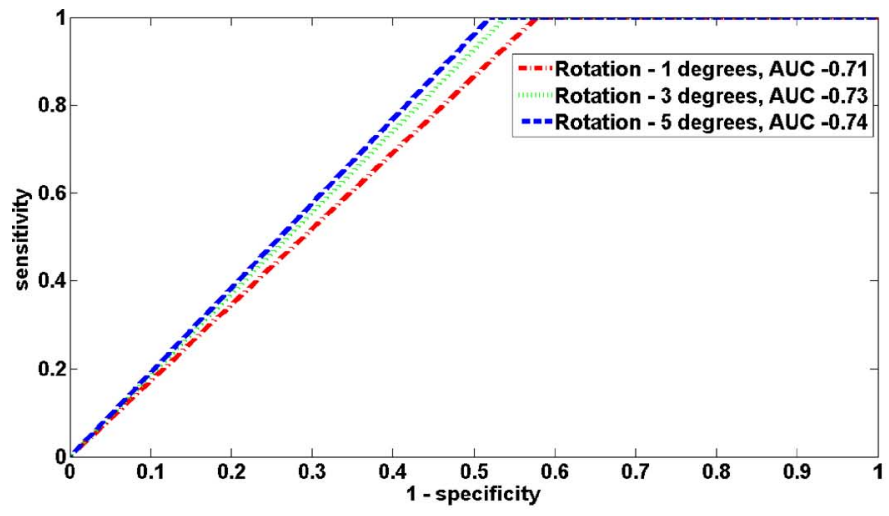

(a)

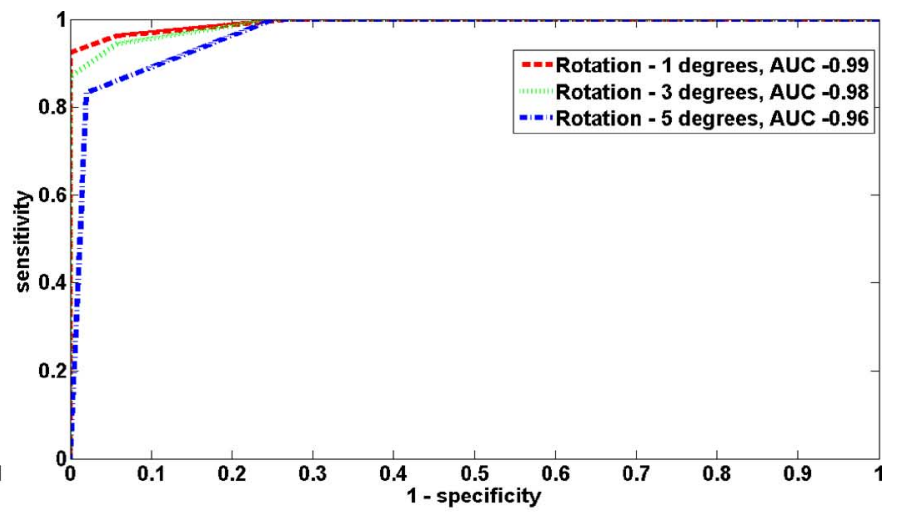

(b)

Fig. 10. ROC curves for the detection of DME from DMED dataset. (a) ROC curve for DMED—Gaussian. (b) ROC curve for DMED—PCA.

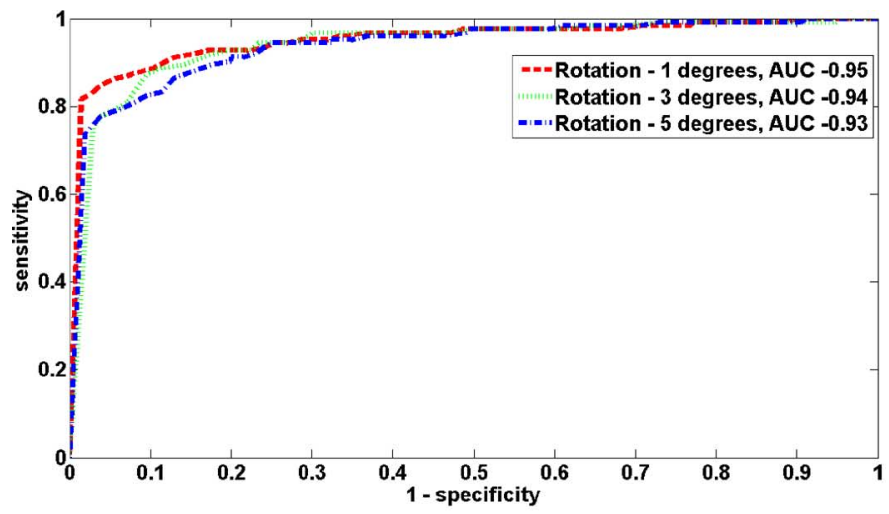

(a)

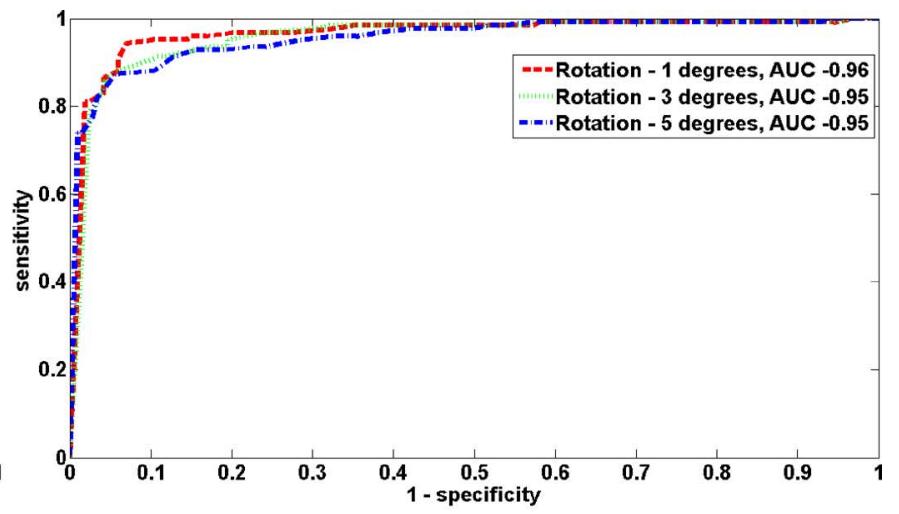

(b)

Fig. 11. ROC curves for the detection of DME from MESSIDOR dataset. (a) ROC curve for MESSIDOR—Gaussian. (b) ROC curve for MESSIDOR—PCA.

TABLE I

Classification Performance of Normal and Abnormal COLOR RETINAL IMAGES ON THE DMED DATASET

\begin{tabular}{lccc}
\hline \hline Method & Sensitivity $(\%)$ & Specificity $(\%)$ & AUC \\
\hline$[1]$ & 100 & $\tilde{1} 9$ & 0.86 \\
Proposed & 100 & 74 & 0.99 \\
\hline \hline
\end{tabular}

for the severe or immediate referral (IR) cases over that in [25] which uses a global approach based on a visual dictionary. An aggregate performance of $70 \%$ specificity with $98 \%$ sensitivity is observed. Since a large number of normal images are present in the test set, we select a point on the curve where specificity is slightly higher at $90 \%$, with sensitivity of $95 \%$ so that fewer false positives occur in disease severity stratification. The corresponding AUC for the dataset is 0.96 . For the IR cases, a $97 \%$ specificity at $100 \%$ sensitivity is observed with an AUC of 0.99 . The best performance is achieved for a rotation step of $1^{\circ}$ with the PCA DD classifier. The same combination of rotation step and classifier is used for cross-validation analysis next.

2) Cross-Validation Analysis: In order to ensure that the result of DME assessment is not biased due to the training set used, a 10-fold cross-validation performance analysis was done. The images in the training and the testing sets were divided into 10 equal partitions. PCA DD was used for the abnormality detection task due to its better performance compared to Gaussian DD in the earlier experiments. A PCA DD classifier was trained
TABLE II

Classification Performance of NoRmal and AbNormal COLOR Retinal IMAGES ON THE MESSIDOR DataseT

\begin{tabular}{lccc}
\hline \hline Method & Sensitivity(\%) & Specificity(\%) & AUC \\
\hline$[25](I R)$ & 100 & 88 & 0.98 \\
Proposed (IR) & 100 & 97 & 0.99 \\
Proposed (all 400 images) & 95 & 90 & 0.96 \\
\hline \hline
\end{tabular}

TABLE III

Ten-Fold Cross-Validation Performance on Five Datasets

\begin{tabular}{lcc}
\hline \hline Dataset & Avg AUC & Std Dev AUC \\
\hline DMED & 0.98 & 0.02 \\
MESSIDOR & 0.96 & 0.04 \\
MESSIDOR(IR) & 0.99 & 0.01 \\
Diaretdb0 \& db1 & 0.96 & 0.05 \\
Combined Dataset & 0.92 & 0.06 \\
\hline \hline
\end{tabular}

with only normal images whereas both normal and abnormal images were used for validation. The rotational motion pattern was generated with a step size of $1^{\circ}$ and the coalescing function was chosen to be maximum function.

The average (arithmetic mean) and standard deviation values of AUC for different datasets are shown in Table III. It can be observed that the average AUC is above 0.92 across the datasets. A superset (labeled as combined dataset) was created by merging the normal and abnormal images from the four 


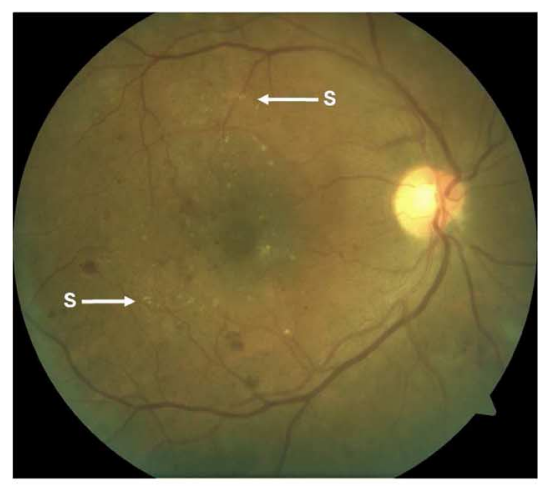

(a)

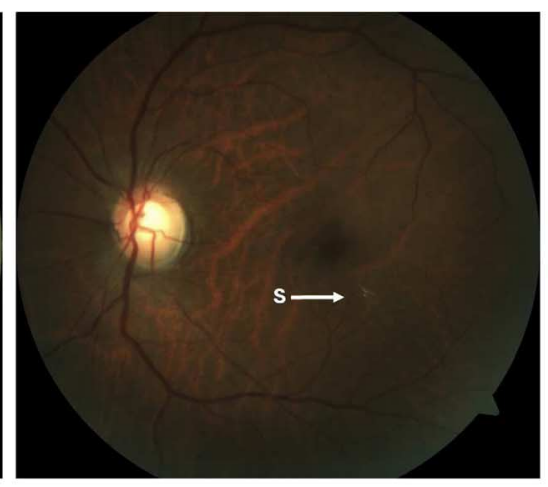

(b)

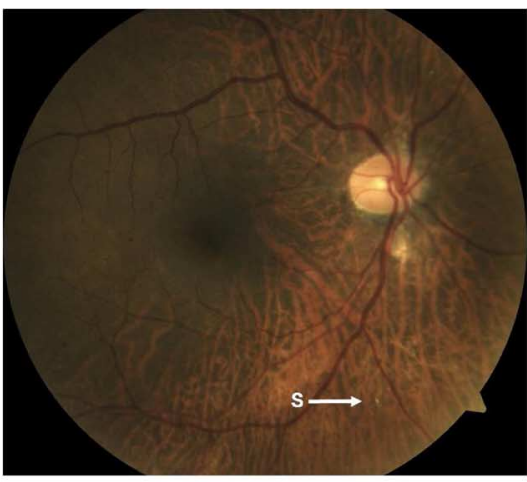

(c)

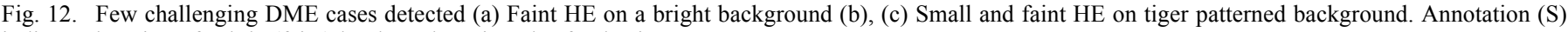
indicates location of subtle (faint) hard exudates in color fundus image.

TABLE IV

SEVERITy ASSESSMENT PERFORMANCE-MESSIDOR

\begin{tabular}{ccc}
\hline \hline $\begin{array}{c}\text { Percentage Threshold (p) } \\
(\% \text { normal asymmetry) }\end{array}$ & $\begin{array}{c}\text { Moderate } \\
\text { accuracy }(\%)\end{array}$ & $\begin{array}{c}\text { Severe } \\
\text { accuracy }(\%)\end{array}$ \\
\hline 0.75 & 51 & 100 \\
0.50 & 53 & 100 \\
0.25 & 81 & 100 \\
\hline \hline
\end{tabular}

public datasets that were considered. This superset consists of 644 images of which 367 were normal and 277 were abnormal images. It should be noted that the average area under ROC curve for immediate referral cases in the MESSIDOR dataset is consistently high at 0.99 compared to the method proposed in [25].

\section{Classification of Macular Edema Cases}

Finally, we studied the effect of the threshold (T) (on rotational symmetry metric) in severity assessment. The results are presented in Table IV for different threshold settings on the images detected as abnormal for MEDDIDOR dataset. The threshold is expressed as a percentage $(p)$ of the symmetry measure $S$ of normal ROIs used in the abnormality detection task. It can be seen that the classification accuracy is high when the value of $p$ is at $25 \%$ of the $S$ value for normals. The classification accuracy for the moderate class falls as the value of $p$ is increased to $75 \%$. This implies that $S$ of the normal ROI is sensitive to intensity variations but does not affect the classification accuracy of severe cases.

\section{CONCLUDING REMARKS}

We have proposed and evaluated a method for DME detection and assessment. The significant contributions of this work are: 1) a hierarchical approach to the problem, 2) a novel representation for the first level, to classify an image as normal/abnormal (containing HE), and 3) a rotational asymmetry measure for the second level, to assess the severity of risk of DME. The novel representation captures the global image characteristics. Such global features have not been used successfully earlier for HE detection. In the first level, a supervised technique based on learning the image characteristics of only normal patients is used for detecting the abnormal cases pertaining to HE.
This approach has the inherent advantage of reducing the effort of building a CAD system by removing the need for annotated (at the lesion level) abnormal images. Such annotations are required for both supervised and unsupervised classification schemes in order to find suitable system parameters for detection. The approach facilitates separating the normal patients from those showing disease symptoms, as practiced in DR screening [9]. There is no need for either preprocessing the original images or postprocessing the results, to handle the false alarms due to variability observed across color fundus images. This is due to the proposed global features. The proposed method is shown to be effective in detecting DME for challenging cases (see Fig. 12). For the fundus image in Fig. 12(a), we can observe that HE manifest as faint lesions whereas in Fig. 12(b), faint HE can be seen over a complex background.

In the second level, the severity of the abnormality is assessed by analyzing the rotational asymmetry of the macular region in retina. This level facilitates the decision to recommend a patient to a medical expert, based on the proximity of HE to the center of macula. The proposed methodology enhances the existing DR screening infrastructure by helping automate the detection and assessment of DME. In this work we have assumed an image to be normal if it has no lesions. Expanding the normal class to include non-DME lesions does not compromise the system's performance as the non-DME lesions are generally dark or bright and diffuse. Consequently, they are well separated in the feature space from HEs. This was confirmed experimentally.

\section{ACKNOWLEDGMENT}

Color fundus images in the MESSIDOR dataset were kindly provided by the Messidor program partners, see (http://messidor.crihan.fr). DMED or HEI-MED images were provided at http://vibot.u-bourgogne.fr/luca/heimed.php. Diaretdb0 and Diaretdb1 dataset were provided at http://www2.it.lut.fi/project/ imageret/

\section{REFERENCES}

[1] L. Giancardo, F. Meriaudeau, T. P. Karnowski, Y. Li, K. W. Tobin, Jr., and E. Chaum, "Automatic retina exudates segmentation without a manually labelled training set," in Proc. 2011 IEEE Int. Symp. Biomed. Imag: From Nano to Macro, Mar. 2011, pp. 1396-1400. 
[2] L. Giancardo, F. Meriaudeau, T. Karnowski, K. Tobin, E. Grisan, P. Favaro, A. Ruggeri, and E. Chaum, "Textureless macula swelling detection with multiple retinal fundus images," IEEE Trans. Biomed. Eng., vol. 58, no. 3, pp. 795-799, Mar. 2011.

[3] M. R. Hee, C. A. Puliafito, C. Wong, J. S. Duker, E. Reichel, B. Rutledge, J. S. Schuman, E. A. Swanson, and J. G. Fujimoto, "Quantitative assessment of macular edema with optical coherence tomography," Arch. Ophthalmol., vol. 113, no. 8, pp. 1019-1029, Aug. 1995.

[4] J. Davidson, T. Ciulla, J. McGill, K. Kles, and P. Anderson, "How the diabetic eye loses vision," Endocrine, vol. 32, pp. 107-116, Nov. 2007.

[5] C. P. Wilkinson, F. L. Ferris, R. E. Klein, P. P. Lee, C. D. Agardh, M. Davis, D. Dills, A. Kampik, R. Pararajasegaram, and J. T. Verdaguer, "Proposed international clinical diabetic retinopathy and diabetic macular edema disease severity scales," Am. Acad. Ophthalmol., vol. 110, no. 9 , pp. $1677-1682$, Sep. 2003.

[6] R. F. N. Silberman, K. Ahlrich, and L. Subramanian, "Case for automated detection of diabetic retinopathy," Proc. AAAI Artif. Intell. Development (AI-D'10), pp. 85-90, Mar. 2010.

[7] M. Verma, R. Raman, and R. E. Mohan, "Application of tele ophthalmology in remote diagnosis and management of adnexal and orbital diseases," Indian J. Ophthalmol., vol. 57, no. 5, pp. 381-384, Jul. 2009.

[8] M. D. Abramoff, M. Niemeijer, M. S. Suttorp-Schulten, M. A. Viergever, S. R. Russell, and B. van Ginneken, "Evaluation of a system for automatic detection of diabetic retinopathy from color fundus photographs in a large population of patients with diabetes," $J$. Diabetes Care, vol. 31, no. 2, pp. 193-198, Nov. 2007.

[9] S. Philip, A. Fleming, K. Goatman, S. Fonseca, P. McNamee, G. Scotland, G. Prescott, P. F. Sharp, and J. Olson, "The efficacy of automated disease/no disease grading for diabetic retinopathy in a systematic screening programme," Br. J. Ophthalmol., vol. 91, no. 11, pp. 1512-7, Nov. 2007

[10] H. Jaafar, A. Nandi, and W. Al-Nuaimy, "Detection of exudates in retinal images using a pure splitting technique," in Proc. Annu. Int. Conf. IEEE Eng. Med. Biol. Soc. (EMBC), Aug. 2010, pp. 6745-6748.

[11] P. C. Siddalingaswamy and K. G. Prabhu, "Automatic grading of diabetic maculopathy severity levels," in Int. Conf. Syst. Med. Biol. (ICSMB), Dec. 2010, pp. 331-334.

[12] C. I. Sanchez, M. Garca, A. Mayo, M. I. Lopez, and R. Hornero, "Retinal image analysis based on mixture models to detect hard exudates," Med. Image Anal., vol. 13, no. 4, pp. 650-658, Aug. 2009.

[13] A. Osareh, M. Mirmehdi, B. Thomas, and R. Markham, "Automated identification of diabetic retinal exudates in digital colour images," $\mathrm{Br}$. J. Ophthalmol., vol. 87, pp. 1220-1223, Oct. 2003.

[14] T. Walter, J.-C. Klein, P. Massin, and A. Erginay, "A contribution of image processing to the diagnosis of diabetic retinopathy-detection of exudates in color fundus images of the human retina," IEEE Trans. Med. Imag., vol. 21, no. 10, pp. 1236-1243, Oct. 2002.

[15] H. Wang, W. Hsu, K. G. Goh, and M. L. Lee, "An effective approach to detect lesions in color retinal images," in Proc. IEEE Conf. Comput. Vis. Pattern Recognit., 2000, vol. 2, pp. 181-186.

[16] W. Hsu, P. Pallawala, M. L. Lee, and K.-G. A. Eong, "The role of domain knowledge in the detection of retinal hard exudates," in Proc. 2001 IEEE Comput. Soc. Conf. Comput. Vis. Pattern Recognit. (CVPR 2001), 2001, vol. 2, pp. II-246-II-251.

[17] C. Sanchez, A. Mayo, M. Garcia, M. Lopez, and R. Hornero, "Automatic image processing algorithm to detect hard exudates based on mixture models," in Proc. 28th Annu. Int. Conf. IEEE Eng. Med. Biol. Soc., Sep. 2006, pp. 4453-4456.

[18] Y. Hatanaka, T. Nakagawa, Y. Hayashi, Y. Mizukusa, A. Fujita, M. Kakogawa, K. K. M. D. , T. Hara, and H. Fujita, "Cad scheme to detect hemorrhages and exudates in ocular fundus images," in Proc. SPIE Med. Imag. 2007: Comput.-Aided Diagn., Mar. 2007, vol. 6514, pp. 2M1-2M8.
[19] S. Ravishankar, A. Jain, and A. Mittal, "Automated feature extraction for early detection of diabetic retinopathy in fundus images," in IEEE Conf. Comput. Vis. Pattern Recognit., Jun. 2009, pp. 210-217.

[20] A. Osareh, B. Shadgar, and R. Markham, "A computational-intelligence-based approach for detection of exudates in diabetic retinopathy images," IEEE Trans. Inf. Technol. Biomed., vol. 13, no. 4, pp 535-545, Jul. 2009.

[21] H. Li and O. Chutatape, "A model-based approach for automated feature extraction in fundus images," in Proc. 9th IEEE Int. Conf. Comput. Vis., Oct. 2003, vol. 1, pp. 394-399.

[22] M. Garcia, R. Hornero, C. Sanchez, M. Lopez, and A. Diez, "Feature extraction and selection for the automatic detection of hard exudates in retinal images," in Proc. 29th Annu. Int. Conf. IEEE Eng. Med. Biol. Soc., Aug. 2007, pp. 4969-4972.

[23] K. Ram and J. Sivaswamy, "Multi-space clustering for segmentation of exudates in retinal color photographs," in Proc. Annu. Int. Conf. IEEE Eng. Med. Biol. Soc., Sep. 2009, pp. 1437-1440.

[24] A. Rocha, T. Carvalho, S. Goldenstein, and J. Wainer, Points of interest and visual dictionary for retina pathology detection Inst. Comput., Univ. Campinas, Tech. Rep. IC-11-07, Mar. 2011.

[25] C. Agurto, V. Murray, E. Barriga, S. Murillo, M. Pattichis, H. Davis, S. Russell, M. Abramoff, and P. Soliz, "Multiscale am-fm methods for diabetic retinopathy lesion detection," IEEE Trans. Med. Imag., vol. 29, no. 2, pp. 502-512, Feb. 2010.

[26] N. P. Ward, S. Tomlinson, and C. J. Taylor, "Image analysis of fundus photographs. the detection and measurement of exudates associated with diabetic retinopathy," Ophthalmology, vol. 96, no. 1, p. 86-6, Jan. 1989.

[27] W. Huan, H. Wynne, and L. M. Li, "Effective detection of retinal exudates in fundus images," in Proc. 2nd Int. Conf. Biomed. Eng. Informat., Oct. 2009, pp. 1-5.

[28] J. Singh, G. D. Joshi, and J. Sivaswamy, "Appearance-based object detection in colour retinal images," in Proc. Int. Conf. Image Process., 2008, pp. 1432-1435.

[29] G. D. Joshi, J. Sivaswamy, K. Karan, and S. R. Krishnadas, "Optic disk and cup boundary detection using regional information," in Proc. Int. Conf. Image Process., 2010, pp. 948-951.

[30] J. V. Stone, "Object recognition using spatiotemporal signatures," $V i-$ sion Research, vol. 38, no. 7, pp. 947-951, Apr. 1998.

[31] S. Cho, Y. Matsushita, and S. Lee, "Removing non-uniform motion blur from images," in Proc. IEEE 11th Int. Conf. Comput. Vis., Oct. 2007, pp. 1-8.

[32] S. Nishida, J. Watanabe, I. Kuriki, and T. Tokimoto, "Human visual system integrates color signals along a motion trajectory," Current Biol., vol. 17, no. 4, pp. 366-372, Feb. 2007.

[33] K. S. Deepak, G. D. Joshi, and J. Sivaswamy, "Content-based retrieval of retinal images for maculopathy," in Proc. 1st ACM Int. Health Inf. Symp., 2010, pp. 135-143.

[34] MESSIDOR, Jun. 2011 [Online]. Available: http://messidor.crihan.fr/ index-en.php

[35] DIARETDB0, DIARETDB0: Evaluation database and methodology for diabetic retinopathy algorithms May 2007 [Online]. Available: http://www2.it.lut.fi/project/imageret/diaretdb0/

[36] DIARETDB1, May 2009, DiaRetDB1: Diabetic retinopathy database and evaluation protocol [Online]. Available: http://www2.it.lut.fi/ project/imageret/diaretdb1/ 